\title{
The Outcome of Midwife-Mother Relationship in Delivery Room: A Qualitative Content Analysis
}

\author{
Mahtab Attarha1, Zohre Keshavarz ${ }^{2 *}$, Maryam Bakhtiari' ${ }^{3}$, Mehri Jamilian ${ }^{4}$ \\ ${ }^{1}$ Department of Reproductive Health \& Midwifery, School of Nursing and Midwifery, Beheshti University of \\ Medical Sciences, Tehran, Iran \\ ${ }^{2}$ Department of Reproductive Health \& Midwifery, School of Nursing and Midwifery, Shahid Beheshti \\ University of Medical Sciences, Tehran, Iran \\ ${ }^{3}$ Department of Clinical Psychology, Shahid Beheshti University of Medical Sciences, Tehran, Iran \\ ${ }^{4}$ Department of Gynecology, School of Medicine, Arak University of Medical Sciences, Arak, Iran \\ Email: "keshavarzzohre@yahoo.com, *z.keshavarz@sbmu.ac.ir
}

Received 20 January 2016; accepted 23 February 2016; published 26 February 2016

Copyright (C) 2016 by authors and Scientific Research Publishing Inc.

This work is licensed under the Creative Commons Attribution International License (CC BY). http://creativecommons.org/licenses/by/4.0/

c) (i) Open Access

\begin{abstract}
Effective communication and emotional support of parturient women improve the outcome of childbirth. Reduction in Duration of labor, cesarean rate, use of anesthesia, and 5 minute Apgar Score less than 7 can achieve. This study was a part of a large mixed method study during 2013-2014. In this qualitative study, in-depth interviews were conducted with 32 participants (16 mother and 16 midwife), using semi-structured interviews. Participants were recruited by purposive sampling with maximum diversity in terms of age, occupation, education and etc. Educational hospital of Arak University of Medical Sciences was considered as the setting. Each interview was conducted at a private and convenient location and took about 30 to 45 minutes. Interviews were audio taped and transcribed verbatim. Conventional content analysis was done for data analysis. Interviews continued until data saturation was obtained. Data were coded in MAXQDA software (version 11). The main category emerged as "outcome" of midwife-mother relationship. Facilitating childbirth, positive experience, mental health promotion and improvement in quality of life were derived as subcategories. Good midwife-mother relationship could promote positive outcome in labour. Results could assist midwives in providing holistic quality care to mothers during labour, thus providing positive consequence in child birth. Also, results could provide a framework and guidance for policymakers to create appropriate context for the midwife-mother relationship in maternity care.
\end{abstract}

${ }^{*}$ Corresponding author.

How to cite this paper: Attarha, M., Keshavarz, Z., Bakhtiari, M. and Jamilian, M. (2016) The Outcome of Midwife-Mother Relationship in Delivery Room: A Qualitative Content Analysis. Health, 8, 336-343.

http://dx.doi.org/10.4236/health.2016.84035 


\section{Keywords}

\section{Midwife-Mother Relationship, Content Analysis, Outcome, Delivery Room}

\section{Introduction}

When health professionals have communication skills, countless positive results for the patients, such as reduced symptoms, pain, anxiety, sense of guilt, increased peace of mind, acceptance and adjustment to their disease, cooperation with the medical team, improved blood pressure and glucose [1], increased effectiveness of training and the prevention of medical errors can be seen [2]. Studies conducted on the doctor-patient interaction confirm that communication goes beyond the exchange of information. Interventions that encourage doctors to acquire the skill of conveying knowledge and involving patients in consultations and decision-making were improved patients' physical and mental health indicators [3] [4]. Good interaction is key to improving health outcomes and eliminating the need for additional care, reducing hospital costs, litigation, improving patient-oriented care and patient satisfaction [5]. The patient's ability to freely express his views, feelings concerns and be involved in the decision-making process and his own treatment leads to better outcomes and a greater sense of satisfaction [6]. The UK Ministry of Health has announced intentions to teach new communication skills to all NHS professionals [7]. These plans are partly in response to the growing evidence from researcher suggesting that the quality of the interaction between patients and their careers has dramatic effects on various aspects of clients' health [3]. Maternity care is an area of health care in which optimal communication is highly emphasized. Midwife is supporter, assister, respecter and comfort to mother during childbirth [8]. Adopting a communicative approach promote self-empowerment in women and allows them to decisions making about their health care [9]. Midwife's relationship with mother is often a spiritual catalyst, emotional growth and transformation for the mother [10]. Low-risk women who give birth with assist of a midwife, experience countless positive outcomes, such as an improved sense of self-confidence [11] reduced hospital stay, LBW and labor interventions [10].

The quality of midwifery care has mostly influenced on childbirth outcome. The midwives' performance and measures during this crucial time, not only affect the mother's mental and emotional health, but also affect neonatal health significantly [12]. The trust between the mother and the careers, the comfortable feeling of being surrounded by loving people who will stay by her, cause positive childbirth experience [13]. From the mothers' view, the main factor involved in having an enjoyable experience of childbirth is receiving the full support of a midwife. Cross-sectional studies conducted on groups of women from different cultural backgrounds, including Indian, Jamaican and Mexicans, have confirmed this finding [14]. An effective communication and an ongoing emotional support improve birth outcome, such as reduced duration of labor, cesarean rate, use of anesthesia, operative delivery and 5 minute Apgar less than 7 [15].

Although, the important role of effective communication in maternity care is illuminated, yet midwife-mother relationship is unfavorable. In 1992, the Ministry of Health in UK and Wales recognized the importance of optimal communication in maternity care and realized that in many cases the relationship was not as good as it should be [16]. The gap in communication leads to poor quality of care, deterioration of health outcomes and likely dissatisfaction of the health care providers. Previous studies have shown that relationship problems between pregnant women and care givers are the main cause of complaint and most of the women are unhappy with the quality and quantity of information that they receive [13]. Patient dissatisfaction with health care providers' relationship may be one of the main causes of medical malpractice.

Few studies have examined the strategies adopted to improve communications between women and their health care providers. Many studies are conducted about physician-patient relationship, but there are few studies about midwife-mother relationship. Regarding the inadequate knowledge about health communication behavior; exploring the experiences of mothers in delivery room and consequences of the effective relationships between midwife-mother; this study was conducted using a qualitative approach. We need to clarify the outcome of effective communication between midwife and mother by naturalistic approach and in-depth interviews. Thus, we interviewed a group of midwives and mothers to determine their experiences and beliefs about outcomes' of the relationship. 


\section{Methods}

\subsection{Study Location and Participants}

This research was a part of a larger mixed method study which was carried out in Iran during 2012-2014. Participants consisted of 16 mothers hospitalized in post-partum ward and 16 midwifes employed in delivery room. This study was carried out from August 2013 until April 2014 in an educational hospital of Arak University of Medical Sciences in Iran. Iranian women who had normal vaginal delivery and midwives who employed in labour were invited to participate in this study. They spoke Persian fluently.

\subsection{Study Design}

This study was a qualitative study with the aim of exploring of midwife-mother relationship in delivery room using a conventional content analysis approach. A qualitative approach identifies the perspectives of the participants and reveals their characteristics and experiences [17].

\subsection{Study Procedures}

After ethics approval (SBMU2.REC.1394.87) the recruitment process started. Data was obtained from 32 semistructured interviews. Participants were 16 mothers and 16 midwives which were recruited by purposeful sampling with maximum diversity. Educational hospital of Arak University of Medical Sciences was considered as the setting. Each interview was conducted at a private and convenient location and took about 30 to 45 minutes. Interviews were audio taped and transcribed verbatim. Interviews continued until data saturation was obtained. The interview was started with open-ended questions and was continued with probing and follow upping questions. The primary interview guide questions were:

Please explain about your feelings in delivery room?

What was your experience in delivery room about midwife-mother relationship?

Does the relationship had any advantages or disadvantages?

As ideas emerged, the interviewer asked more focused questions. An occasional probe such as "can you tell me a little more about this” which helped to elicit more information about the participants.

Written narratives were found to be the most appropriate data collection manner in order to encourage the participants to describe their experience and consequence of the effective midwife-mother relationship for the present study.

\subsection{Data Analysis}

Data was analyzed using conventional content analysis method, guided by Graneheim and Ludman [18]. Interviews were completely audio-taped and transcribed verbatim. Data were coded in MAXQDA software (version 11). The condensed meaning units were abstracted and labelled with codes. Then the codes were organized into subcategories and then categories derived based on comparisons regarding their similarities and differences.

\subsection{Validity of the Study}

In order to ensure the credibility of research data, participants' checking was carried out. During the interview, the interviewee's words were fed-back to participants to confirm them. On the other hand, the codes extracted from the 4 interviews were shown to the respective participants and corrected under their supervision. Other methods such as prolonged engagement, sufficient time allocation and good communication were utilized.

In order to reach transferability, detailed and thick data description was applied so that other researchers can have a full understanding of research steps.

In order to achieve conformability, two experts in the field of qualitative research and reproductive health were asked to review the reports and manuscripts and present their findings. Very high similarity was found in conclusions.

In order to evaluate credibility, data was presented to an external researcher and it was found that he concluded the same as the relevant researcher and as such credibility and dependability of data was achieved. 


\subsection{Ethical Consideration}

Both, the Research deputy and Ethics Committee of nursing and midwifery School of Shahid Beheshti University of medical sciences and Arak University of Medical Sciences approved this study. The aim of the study was explained in detail to the participants. Informed consent were obtained from the participants to perform and record the interviews. The following information was given to the participants: the voluntary nature of the participation and withdraw from the study at any time they wished, their right to privacy and confidentiality.

\section{Results}

\subsection{Study Population}

A total of 16 midwives and 16 mothers participated in this study. The midwives were 23 - 45 years of age. Eight midwives were married. Their job experiences range were 2 months to 20 years. Fifteen midwives had BA and one had MA degree in midwifery. Ten midwives had tenure and six had contract employment.

Mothers were 19 - 36 years of age. To make ensure of maximum variation, the participants were selected from various ages, parities, educational levels, location and socio-economic backgrounds in order to get diversity in experiences and perceptions. Four mothers had one delivery, six mothers had two, four mothers had three and two mothers had four. Four mothers had primary school, three mothers had secondary school, six had high school and three had BA degrees. Eleven women were urban dwellers.

\subsection{Category and Subcategory}

In this qualitative research, thematic analysis revealed that: The main category "outcome” consists of four subcategories including: Facilitating childbirth, positive experience, mental health promotion and improvement in quality of life (Table 1).

Effective midwife-mother relationship leads to a positive childbirth's outcomes and promoting maternal and neonatal health.

\subsubsection{Facilitating Childbirth}

Participants had stated that considering parturient condition and comforting help, alleviate her concerns and has a significant role in pain relief and labour progress.

A midwife explained, "It is undeniable that a strong interaction affects the delivery process and the midwife comforting the mother is highly helpful for the labour progress. When the mother realizes that there is someone

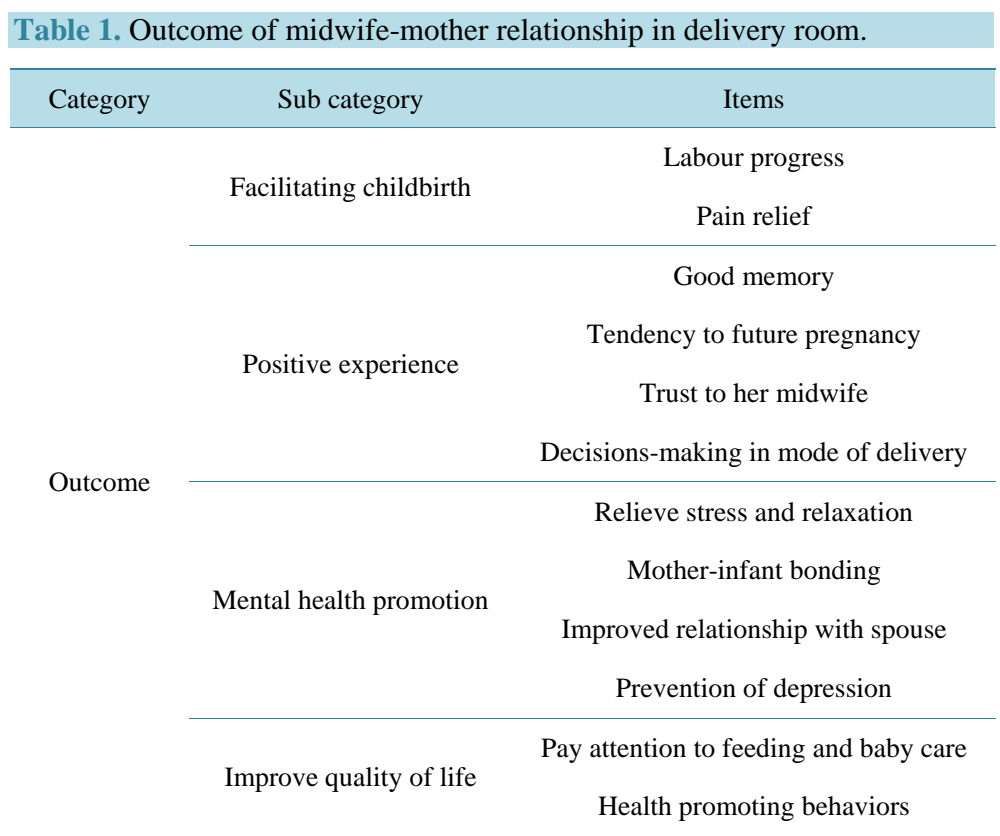


who understands her, she feels comforted and her pain becomes more tolerable, which then affects her dilation and effacement, but more dilation, of course" (M2, 29 years old, with 5 years of work experience). One mother asserted, "When they explain things to with common curtesy so you won't be disheartened ... it enables you to have a more comfortable delivery. They should tell you what to do to be decrease pain and deliver the baby more quickly" (W2, 28 years old, second delivery).

\subsubsection{Positive Experience}

Relational Continuity is a key concept in creating a positive and enjoyable experience of childbirth and allows the midwife to provide the mother holistic care. Moreover, this leads to positive outcomes such as the desire to become pregnant again, trust to her midwife and decisions-making about mode of delivery.

For example a mother explained, "When a mother realizes that the midwife is treating her well and is listening to her, she gives birth more comfortably and gets through labor with a good memory. So she feels more comfortable becoming pregnant again, because it's good and easy and makes you feel better than when you are shouted at and scolded about not trying hard enough" (W3, 36 years old, third delivery).

Good interaction assists to create trust and better mother's cooperation with the midwife. The expression of one midwife discloses this sense, "When communication is established, the patient begins to trust us and then cooperates much better. After shift changes, when the new midwives introduce themselves, the patients come and ask for a certain midwife, or sometimes they hold our hands and ask us to deliver their child ourselves; this just shows how good our relationship with them has been" (M5, 42 years old, 18 years of work experience).

\subsubsection{Promotion of Mental Health}

Four results of mental health promotion include: Relieve stress and relaxation; mother-infant bonding; improved relationship with spouse; prevention of depression.

Majority of participants believed that a good interaction with mother was key to reduce her stress, and make her feel calm. One midwife argued, "The midwife-mother interaction creates a calmer mother and facilitates a more comfortable delivery, because it has a very positive mental impact on the mother" (M4, 36 years old, 9 years of work experience). One mother added, "With the proper attitude and behavior, the stress experienced in the delivery room decreases; but a bad attitude adds to delivery room stress surely increases the mother's stress" (W11, 26 years old, first delivery).

Establishing a proper verbal communication and talking with mother about her child that will be born in a few hours, help to create an emotional and affective bonding between her and the infant. Thereby a better relationship with the spouse as well. A midwife said, "When you talk to her about the soon-to-be-born baby and how she should be more resilient as the mother, when you tell that she is no longer just one person, but two, and you try to support her, they take you much more seriously, and some of them even react to you the second you take the baby to their bed after delivery. For example, when you comment on the baby's beautiful face or hair, they take greater interest in what you say" (M15, 28 years old, 5 years of work experience).

Mothers also mentioned that a better emotional relationship with their child and husband and feeling less depressed are the consequences of an effective midwife-mother relationship. "When you are treated with kindness and compassion, you don't get nervous anymore or yell at your kid and you treat your husband well too" (W7, 32 years old, second delivery).

Understanding and assuring the mothers and giving them the opportunity to express their concerns are effective in making the mother feel calm and reducing postpartum depression. One midwife commented, "The assurance provided by the midwife to the mother, that there is someone she can talk to and who will understand her, makes her much calmer, and when the mother is calm and composed, she experiences less postpartum depression and can better relate to her child" (M12, 30 years old, 7 years of work experience).

"A mother who is unhappy with her childbirth always remembers the behavior and words of the staff, which have a lasting effect on her. When a mother is nervous, moody and depressed and gets postpartum depression too, she can no longer look after her child properly" (W15, 29 years old, fourth delivery).

The last outcome reported for the midwife-mother interaction involved an improved quality of life and the adoption of health-promoting behaviors. A good midwife-mother relationship increased the mothers' knowledge about maternal and neonatal health care, especially when they lived away from their family, and encouraged the adoption of health-promoting behaviors such as preventing genital and episiotomy infections. One mother explained, "When a mother doesn't know anything about raising children and taking care of a newborn, how to 
change the baby's diaper, how to treat the baby when he is sick and how to give him medications, and when there are no experienced older parents around to tell her how to take care of these things and how to take care of her own stitches too, then the information provided by the midwife will be useful" (W1, 26 years old, first delivery). One midwife asserted, "The training we provide the mothers with has been useful and will undoubtedly be remembered, and even if she goes to a clinic and they tell her otherwise, she will respond by saying that my midwife said this and now you're telling me differently. So the training the midwife has given her has been effective on care for her baby. She listens if you tell her that her baby will be mentally disabled if you don't take her to a doctor when she gets jaundice" (M11, 38 years old, 17 years of work experience).

\section{Discussion}

This study highlights how positive outcome as a result of midwife-mother relationship is formed in delivery room.

Midwifery care support and improves adaptation to stresses of childbirth, enabling mother to experience a more comfortable labour with less anxiety. Delivery, the environment and procedures of it all contribute to the mother's stress [14].

The results of the present study confirmed that the midwife-mother relationship in delivery room is essential to facilitating childbirth. A study conducted by Pascalo Bonaro \& Kroger (2004) showed that supporting the mother during childbirth mitigates the intensity of her pain, facilitates childbirth, shortens labour and improves positive aspect of the experience of birth and the need for analgesics was reduced by $28 \%$ in the supported group compared to the control group [19]. Several studies have shown numerous physiological and psychological benefits for an effective and supportive midwife-mother interaction, which leads to a reduced intensity of pain and thereby a significant reduction in the need for analgesics and ultimately better pregnancy outcomes [20]-[23]. Quoting Rosen, Ahmadi Afshar writes, "Creating a close interaction, building trust and providing an effective ongoing support during labor help stopping the fear-stress-pain cycle thus reduce catecholamine secretion and creating mother' relaxation, thereby improving uterine contraction, reducing the intensity of pain, duration of labor and accelerating physiologic childbirth" [21].

Following the experience of childbirth, women can grow and change in different ways which they give birth regardless of the contexts of their child bearing. It seems that, in shared relationship with a midwife, her role is to make future mothers mentally and emotionally prepared for childbirth through listening to her with genuine interest.

Women's positive experience of relational continuity through pregnancy, labor and postnatal was one of the main consequence of effective relationship. Hunter et al. (2008) highlight this important viewpoint. The obvious factors in maternity care are the clinical results and technology, whilst the relationships that keep it all together are an unseen factor in the final outcome [23].

Dahlberg writes, "Midwifery care is the best method of creating a positive experience of childbirth for mothers, and midwife's communication skills, knowledge, understanding and their presence are vital to this experience" [24]. Several studies have also confirmed that women who have a good interaction with their midwife feel better about themselves, childbirth and their baby [15] [23] [25] [26]. Trust to the midwife may be the result of a continuous interaction during the process of childbirth [24]. Thomson \& Downe (2010) have emphasized that the midwife-mother interaction is essential to achieve a positive experience of childbirth. According to continuity of care model, interaction is based on trust, mutuality and respect [27].

Better maternity care, relational continuity and emotional support provided by midwife, lead to mother's mental health promotion. Rossiter showed that the mothers' improved psychological health constitutes one of the important aspects of this interaction. Mothers reported an increased self-esteem, accountability, self-worth, sense of power and self-appreciation along with their reduced stress. They also revealed an increased confidence about their prenatal care, childbirth as normal process, postpartum experiences and parenting. They felt empowered due the relationship and stated that they had learned life lessons from this relationship [28]. Our results are consistent with the results of previous studies [24] [29].

Midwifery care also provides some certain sources of support for future mothers. For instance, providing social support during labor reduces her likelihood of developing postpartum depression [28]. Midwives can act as the health provider of the entire family and detect families that are vulnerable to depression and take preventive measures to help them [30]. 
The midwife-mother relationship involved an improved quality of life and the adoption of health-promoting behaviors. Such as increased the mothers' information about maternal and neonatal health care, especially when they haven't any experience about those.

The results of a similar study showed that care givers' support has positive effects on the quality of life. Support during labor also increases breastfeeding success and creates a strong positive mother-child relationship and reduces the need for interventions. This support includes continuity of care, a good interaction and encouraging the patient [31].

Studies commissioned by the National Health System showed that the quality of the established interaction affects various aspects of the patients' wellbeing. These include knowledge, compliance with the treatment, satisfaction, quality of life, mental health status and other health outcomes [3].

The study's points of strength include using the experiences and viewpoints of mothers and midwives in delivery room regarding midwife-mother relationship. Caution in the generalization is the limitations of all the qualitative studies. The researchers thus made great efforts to improve the rigor of the data. The limitation of the study was the emphasis on the midwife-mother relationship during childbirth process. However, results can be used as the basis for future studies.

\section{Conclusion}

The therapeutic value of an effective relationship should be recognized, as it can help explain the unique contribution of midwifery care. The possibility of relational continuity allows midwives to provide mothers with a high-quality holistic care and helps improve positive labor outcomes. Women should be encouraged to ask questions, be given clear verbal information and be as emotional supported as possible. The results of the present study can provide midwifery policy-makers and planners with a mutual understanding of the proper context in which these interactions can be established. It is evident that the complex issue of interaction requires more extensive investigations to increase understandings of its different features and consequences, especially since it is a less discussed issue. As relationship is an interactive process, further qualitative studies can prove beneficial. Researchers hope that results can provide a strong foundation for future efforts and better understanding the key role of this concept and possible ways for creating, maintaining and strengthening the midwife-mother interaction. The outcomes of midwife-mother relationship can be used as the basis of new courses in midwifery curricula. Future experimental studies are recommended to focus on the assessment of such educational programs and the outcomes in delivery room.

\section{References}

[1] Aghabarary M, Mohammadi, E. and Varvani-Farahani, A. (2009) Barriers to Application of Communicative Skills by Nurses in Nurse-Patient Interaction: Nurses and Patients' Perspective. Journal of Nursing Midwifery Iran University of Medical Sciences, 22, 19-31. (In Persian)

[2] Asemani, O. (2011) A Review of the Models of Physician-Patient Relationship and Its Challenges. Iranian Journal of Medical Ethics and History of Medicine, 5, 44-57. (In Persian)

[3] Rowe, R.E., Garcia, J., Macfarlane, A.J. and Davidson, L.L. (2002) Improving Communication between Health Professionals and Women in Maternity Care: A Structured Review. Health Expectations, 5, 63-83. http://dx.doi.org/10.1046/j.1369-6513.2002.00159.x

[4] Rowe, R., Garcia, J., Macfarlane, A.J. and Davidson, L.L. (2001) Does Poor Communication Contribute to Stillbirth and Infants Death? A Review. Journal of Public Health, 23, 23-34. http://dx.doi.org/10.1093/pubmed/23.1.23

[5] Moslemabadi Farahani, S., Malekzadegan, A., Mohammadi, R. and Hosseini, F. (2005) Effect of the One to One Midwifery Care during Labor on Modes of Delivery. Iran Journal of Nursing, 18, 71-82. (In Persian)

[6] Yeh, J. and Nagel, E.E. (2010) Patient Satisfaction in Obstetrics and Gynecology: Individualized Patient-Centered Communication. Women's Health, 3, 23-32. http://dx.doi.org/10.4137/cmwh.s5870

[7] Hargie, O., Dickson, D., Boohan, M. and Hughes, K. (1998) A Survey of Communication Skills Training in UK Schools of Medicine: Present Practices and Prospective Proposals. Medical Education, 32, 25-34. http://dx.doi.org/10.1046/j.1365-2923.1998.00154.x

[8] Vincent, P. (2002) Baby Catcher: Chronicles of a Modern Midwife. Scribner, New York.

[9] Thachuk, A. (2007) Midwifery, Informed Choice, and Reproductive Autonomy: A Relational Approach. Feminism and Psychology, 17, 39-56. http://dx.doi.org/10.1177/0959353507072911 
[10] Sharpe, M. (2004) Intimate Business: Woman-Midwife Relationships. Dissertation Abstracts International, Ontario.

[11] Parratt, J. and Fahy, K. (2003) Trusting Enough to Be Out of Control: A Pilot Study of Women's Sense of Self during Childbirth. Australian Midwifery, 16, 15-22. http://dx.doi.org/10.1016/s1031-170x(03)80011-9

[12] Hosseini, F. (2005) Effect of the One-to-One Midwifery Care during Labor on Modes of Delivery. Iran Nursing Journal, 18, 71-81. (In Persian)

[13] Sehati, F., Najarzade, M., Seyed Rasooli, E. and Zamn Zade, V. (2009) The Effect of Continuity of Midwifery Care during the Length of Labor and Delivery. Nursing \& Midwifery Journal, 4, 13-18. (In Persian)

[14] Kordi, M., Bakhshi, M. and Tara, F. (2014) The Effect of Continuous Support during Labor on Labor Progress in Primigravida Women. The Iranian Journal of Obstetrics, Gynecology and Infertility (IJOGI), 14, 7-14. (In Persian)

[15] Khadivzadeh, T., Katebi, M.S., Sepehri Shamloom, Z. and Esmaily, H. (2015) Assessment of Midwives’ Communication Skills at the Maternity Wards of Mashhad Teaching Hospitals in 2014. Journal of Midwifery and Reproductive Health, 3, 394-400.

[16] Department of Health (1993) Changing Childbirth: Part I: Report of the Expert Maternity Group. HMSO, London.

[17] Bluff, R. and Holloway, I. (1994) "They Know Best”: Women’s Perceptions of Midwifery Care during Labour and Childbirth. Midwifery, 10, 157-164. http://dx.doi.org/10.1016/0266-6138(94)90046-9

[18] Graneheim, U.H. and Lundman, B. (2004) Qualitative Content Analysis in Nursing Research: Concepts, Procedures and Measures to Achieve Trustworthiness. Nurse Education Today, 24, 105-112. http://dx.doi.org/10.1016/j.nedt.2003.10.001

[19] Pascalo Bonaro, D. and Kroger, M. (2004) Continuous Female Companionship during Child Birth: A Crucial Resource in Time of Stress or Calm. Journal of Midwifery \& Women's Health, 49, 19-27. http://dx.doi.org/10.1016/j.jmwh.2004.04.017

[20] Hunter, L. (2002) Being with Women: A Guiding Concept for the Care of Laboring Women. Journal of Obstetric, Gynecologic \& Neonatal Nursing, 31, 650-657. http://dx.doi.org/10.1177/0884217502239213

[21] Ahmadi Afshar, Z. (2010) Evaluation of the Effect of Continouse Midwifery Support on Pain Intensity in Labour and Delivery. Journal of Rafsanjan University of Medical Sciences, 9, 293-304. (In Persian)

[22] Hodnett, E.D., Gates, S., Hofmeyr, G.J. and Sakala, C. (2013) Continuous Support for Women during Childbirth (Review). Cochrane Database of Systematic Reviews, 5, Article No.: CD003766.

[23] Hunter, L.P. (2009) A Descriptive Study of "Being with Women” during Labour and Birth. Journal of Midwifery \& Women's Health, 54, 111-118.

[24] Dahlberg, U.I.A. (2013) The Woman's Birth Experience-The Effect of Interpersonal Relationships and Continuity of Care. Midwifery, 29, 407-415. http://dx.doi.org/10.1016/j.midw.2012.09.006

[25] Hunter, B. (2006) The Importance of Reciprocity in Relationships between Community Based Midwives and Mothers. Midwifery, 22, 308-322. http://dx.doi.org/10.1016/j.midw.2005.11.002

[26] Nilsson, C. and Lundgren, I. (2009) Women’s Lived Experience of Fear of Childbirth. Midwifery, 25, e1-e9. http://dx.doi.org/10.1016/j.midw.2007.01.017

[27] Thomson, G.M. and Downe, S. (2010) Changing the Future to Change the Past: Women's Experiences of a Positive Birth Following a Traumatic Birth Experience. Journal of Reproductive and Infant Psychology, 28, 102-112. http://dx.doi.org/10.1080/02646830903295000

[28] Rossiter, L. (2008) The Development Midwife-Mother Relationship Questionnaire (MMRQ). PhD Thesis, Faculty of Graduate Studies and Research, University of Regina, Regina.

[29] Sengane, M. (2013) Mothers’ Expectations of Midwives’ Care during Labour in a Public Hospital in Gauteng. Curationis, 36, 1-9. http://dx.doi.org/10.4102/curationis.v36i1.320

[30] Hassan Zahraee, R.F.F., Yazdani, M., Ahmadi, Z. and Bashardoost, N. (2003) Supportive Role of the Midwife in Preventing Post Partum Depression. The Journal of Qazvin University of Medical Sciences, 25, 19-24. (In Persian)

[31] Askari, F., Atarodi, A.T.S., Delshad Noghabi, A. and Sadegh Moghadam, L.R.R. (2010) Women’s Labor Experience: A Phenomenological Study. Ofogh-e-Danesh GMUHS Journal, 16, 39-47. (In Persian) 